\title{
Problems and Prospects in Planetesimal Formation
}

\author{
Eugene Chiang ${ }^{1}$, Ruth Murray-Clay ${ }^{2}$ and Ji-Ming Shi ${ }^{1}$ \\ ${ }^{1}$ Department of Astronomy, UC Berkeley \\ Hearst Field Annex B-20, Berkeley CA 94720, USA \\ email: echiang@astro.berkeley.edu, jmshi@berkeley.edu \\ ${ }^{2}$ Harvard-Smithsonian Center for Astrophysics \\ 60 Garden Street, Cambridge MA 02138, USA \\ email: rmurray-clay@cfa.harvard.edu
}

\begin{abstract}
On the long road from dust to planets there are planetesimals. We review and criticize proposed mechanisms for planetesimal formation, and point out topics for future work.
\end{abstract}

Keywords. planets and satellites: formation, solar system: formation, instabilities, turbulence, Kuiper Belt, minor planets, asteroids, accretion, accretion disks

\section{Introduction}

A popular topic in astronomy classes is tidal disruption: the shredding of a body that comes too close to another. Around black holes, this is called spaghettification. But the reverse process can also occur. Gravitational instability can form stars, planets, and perhaps even planetesimals: the building blocks of planets. The hope is that "spaghettified" dust particles, each no larger than a $\mathrm{cm}$, can collect into regions so dense that they gravitationally collapse into the first-generation planetesimals, having sizes $\gtrsim$ kilometers.

A benchmark density is the Roche density, which is that required for a one-fluid body to resist tidal shear (cf. $\S 4$ ), and which we will approximate by $\rho_{\text {Roche }} \sim M_{*} / a^{3}$, where $M_{*}$ is the mass of the central star and $a$ is the orbital distance. The Roche density is much higher than the gas densities usually attributed to protoplanetary disks; for example, $\rho_{\text {Roche }}$ exceeds the gas density in the minimum-mass solar nebula by $\sim 10^{2}$. The situation for dust is still more daunting. For dust to reach Roche density, the dust-togas ratio $\mu \equiv \rho_{\mathrm{d}} / \rho_{\mathrm{g}}$ must increase by $10^{4}$, from the diffuse interstellar medium value of $10^{-2}$ to $\mu_{\text {Roche }} \sim 10^{2}$. Comparing $\mu_{\text {Roche }}$ to the highest dust-to-gas ratios on Earth (e.g., smoke-filled rooms; Saharan dust storms) is an exercise left for the reader.

This article comments on some recent efforts to understand how such extremely dusty environments might be achieved.

\section{The Streaming Instability and Particle-Particle Sticking}

One way to concentrate particles is through the streaming instability (SI), a two-fluid instability afflicting gas and dust in rotating disks. Youdin \& Goodman (2005) investigated linear wave disturbances in these two frictionally coupled fluids and discovered that there is always an unstable mode that clumps particles aerodynamically. See Jacquet et al. (2011) for a mechanistic explanation.

The SI drives turbulence, and dust clumps within "streaming turbulence" have $\mu$ as large as $\sim 3000$ (Bai \& Stone 2010), well exceeding $\mu_{\text {Roche }}$. Indeed gravitational collapse into bound clumps has been observed in simulations that combine streaming turbulence 
with self-gravity (e.g., Johansen et al. 2012). Unfortunately the degree of clumping is sensitive to particle size, or more accurately, particle stopping time $t_{\text {stop }}$ : the time a particle takes to "come up to speed" in a gas flow. Small particles, easily entrained in gas, have short $t_{\text {stop }}$. The highest values of $\mu$ occur when $t_{\text {stop }}$ is of order the orbital time $\Omega^{-1}$, i.e., the turnover time for the largest eddies in streaming turbulence. Bai \& Stone $(2010)$ found that when $\tau_{\text {stop }} \equiv \Omega t_{\text {stop }} \approx 0.1-1$, streaming turbulence drives max $\mu \sim 3000$, but when $\tau_{\text {stop }} \sim 0.01-0.1, \max \mu \sim 30$. Decreasing $\tau_{\text {stop }}$ decreases $\max \mu$. $\dagger$

Unfortunately, models of particle-particle sticking have yet to robustly produce $\tau_{\text {stop }} \sim$ 1 particles in abundance. As particles grow, their relative velocities increase to the point where collisions result in bouncing or destruction. Standard calculations find max $\tau_{\text {stop }} \sim$ $10^{-3}(\mathrm{~mm}-\mathrm{cm})$ at $1 \mathrm{AU}$ (Zsom et al. 2011). Greater growth is achieved by accounting for the full distribution of relative velocities rather than using rms values; the idea here is that a few lucky bodies can grow to large sizes through a series of low-velocity collisions. Figure 6 of Garaud et al. (2013) impresses: at 1 AU, particles stick to sizes of $10^{2} \mathrm{~m}$ (see also Meru et al., this volume)! What remains to be shown is whether such growth (which is slow because it relies on an improbable sequence of events) proceeds fast enough to overcome the "meter-size barrier": the loss of $\tau_{\text {stop }} \sim 1$ (m-size) bodies to the star from inward drift.

In addition to relying on initial particle sizes that may be unrealistically large, the SI might spawn planetesimals that are too big. Johansen et al. (2012) argued that planetesimals having compact-equivalent sizes of several hundred $\mathrm{km}$ would form from the SI at $\sim 30$ AU. $\ddagger$ Schlichting et al. (this volume) cannot reproduce the size distribution of Kuiper belt objects observed today if the primordial belt were to comprise such large objects - but for a contrary view, see Campo Bagatin \& Benavidez (2012).

\section{Revisiting Goldreich-Ward}

A path to gravitational instability that does not rely on $\tau_{\text {stop }} \sim 1$ particles is vertical settling of grains into a thin dense sheet at the midplane (Goldreich \& Ward 1973). The usual objection is that vertical shear across this "sublayer" triggers the Kelvin-Helmholtz instability (KHI), driving turbulence and halting sedimentation (Weidenschilling 1980). But direct numerical simulations show that the KHI limits itself to the top and bottom surfaces of the sublayer - naturally, since density/velocity gradients are steepest thereleaving dust in the heart of the sublayer free to settle into dense cores (Lee et al. 2010ab; Figure 1). In disks whose bulk (height-integrated) metallicities are enriched over solar by a few, $\mu$ approaches 30 in these cores. Still larger $\mu$ could be attained by adding self-gravity and better resolving the cores spatially.

Thus the classic Goldreich-Ward scenario appears viable insofar as the danger posed by the KHI can be neutralized in disks enriched in metals (see also Weidenschilling 2006; Chiang 2008). Nevertheless, other forms of turbulence threaten. The sublayer tolerates diffusivities no larger than $\alpha \lesssim 3 \times 10^{-7}\left(\tau_{\text {stop }} / 10^{-3}\right)(a / \mathrm{AU})^{4 / 7}$ (Chiang \& Youdin 2010, p509). Streaming turbulence undercuts particle concentration by preventing settling and lowering the average particle density (turbulence extends the wings of the density PDF but lowers the mean).

$\dagger$ Vortices also strongly prefer to concentrate $\tau_{\text {stop }} \sim 1$ particles (Meheut et al., this volume).

$\ddagger$ Our own naive estimate of the mass of bound objects formed by the SI using $\tau_{\text {stop }} \sim 1$ particles is $\sim \rho_{\text {Roche }}(\eta a)^{3}$, with $\eta a \sim\left(c_{\text {gas }} / \Omega a\right)^{2} a$ the largest eddy size, gas sound speed $c_{\text {gas }}$, and Kepler speed $\Omega a$. Our estimate yields a clump mass of $0.05 M_{\oplus}$ at $30 \mathrm{AU}$, of order the mass of the entire Kuiper belt interior to $50 \mathrm{AU}$. 


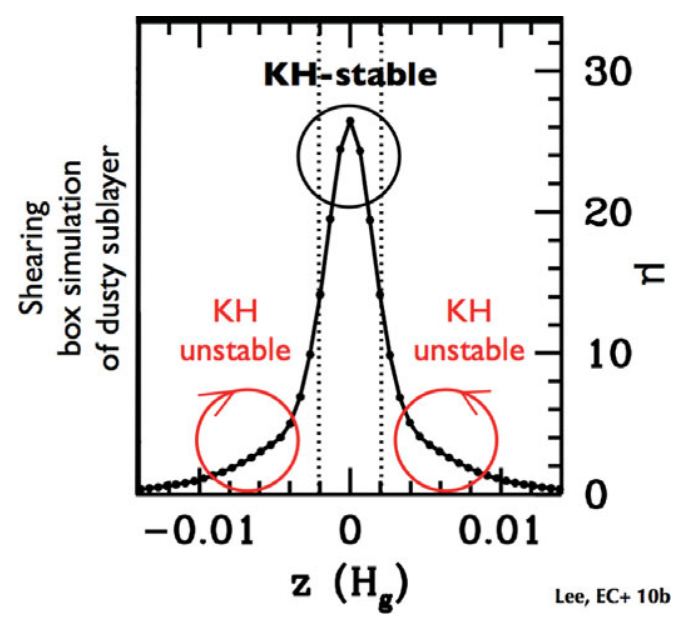

Figure 1. Dust-to-gas volume density ratio $\mu$ vs. height $z$ (in units of gas scale height $H_{\mathrm{g}}$ ) in a settled "sublayer" (figure adapted from Lee et al. 2010b). The bulk disk metallicity (dust-to-gas surface density ratio) is $Z_{\text {disk }}=\Sigma_{\mathrm{d}} / \Sigma_{\mathrm{g}}=0.06=4 \times$ solar. Only the top and bottom surfaces of the sublayer were observed to be Kelvin-Helmholtz $(\mathrm{KH})$ unstable; the core settled freely, and would have settled to still larger $\mu$ than is shown here if the grid resolution were finer.

\section{So You've Reached Roche Density: Now What?}

We have used the Roche density as a benchmark, but it is not much more than that. Attaining Roche density does not guarantee that dust can collapse against gas pressure or rotational shear. Consider a clump of dimension $\ell$ so large that its sound-crossing time $\ell / c>t_{\text {stop }}$. In this "large-clump" regime, the dust-gas mixture acts as a tightly coupled suspension (like milk). Its effective sound speed is $c=c_{\text {gas }} / \sqrt{1+\mu}$, where the square root accounts for how dust adds to the mixture's "mean molecular weight." In this regime, $\ell / c \ll 1 / \sqrt{G \rho_{\text {Roche }}}$ : large Roche-density clumps are Jeans-stable (stabilized by gas pressure; Cuzzi et al. 2008; Shi \& Chiang 2013).

"Small" clumps $\left(\ell / c \ll t_{\text {stop }}\right)$ do not face this difficulty; short-wavelength/ high-frequency sound waves in gas do not couple with dust. Thus small clumps are not supported by gas pressure - but they can still resist collapse because of their finite angular momentum. Although pressure is no longer an impediment, particle collapse speeds are limited by drag to terminal speeds of $v_{\text {term }} \sim f t_{\text {stop }}$, where the self-gravitational acceleration $f \sim G\left(\rho \ell^{3}\right) / \ell^{2} \sim G \rho \ell$. The terminal-speed limit, frustratingly slow, is generic to secular (i.e., dissipative) gravitational instability (GI), and renders collapsing clumps vulnerable to disruption by turbulence (Youdin 2011, see his Figure 4). For self-gravity to defeat rotational shear, $v_{\text {term }} \gtrsim \Omega \ell$ or $\rho \gtrsim \rho_{\text {Roche }} / \tau_{\text {stop }}$ : a truly high bar to clear for small $\tau_{\text {stop }}$. Recent studies of secular GI punt on the obstacle of rotational shear by studying only axisymmetric disturbances (Youdin 2011; Michikoshi et al. 2012). The good news is that axisymmetric modes grow by secular GI no matter what the gas pressure and for any $\rho$, even $\rho \ll \rho_{\text {Roche }}$, but the bad news is that growth plods along at terminal speeds.

\section{Future Directions}

(a) All the problems of planetesimal formation arise because of gas. Perhaps planetesimals form only where gas is severely depleted. The idea seems heretical because gas is required to form giant planets like Jupiter. But maybe the acquisition of gas 
envelopes occurs late in disk evolution, by rocky cores intercepting the last dregs of disk gas transported by viscous accretion across large distances.

(b) The new collisional kernels adopted by Garaud et al. (2013) are promising, but need to be incorporated into a global model that includes radial drift of solids.

(c) How dead are MRI-dead zones, and to what extent can particles settle in such environments (Bai \& Stone 2013; Simon et al., this volume)?

(d) The threat posed by the KHI to Goldreich-Ward can be circumvented for metalenriched disks, but what about the danger presented by the SI? For $\tau_{\text {stop }} \sim 10^{-3}$ and supersolar bulk metallicities, does the SI prevent vertical settling to $\mu \gtrsim 30$ ?

(e) How does secular GI proceed non-axisymmetrically?

We thank P. Hopkins, C. Ormel, R. Rafikov, and A. Youdin for discussions.

\section{References}

Bai, X.-N. \& Stone, J. M. 2010, ApJ, 722, 1437

Bai, X.-N. \& Stone, J. M. 2013, ApJ, 769, 76

Campo Bagatin, A. \& Benavidez, P. G. 2012, MNRAS, 423, 1254

Chiang, E. 2008, ApJ, 675, 1549

Chiang, E. \& Youdin, A. 2010, AREPS, 38, 493

Cuzzi, J. N., Hogan, R. C., \& Shariff, K. 2008, ApJ, 687, 1432

Garaud, P., Meru, F., Galvagni, M., \& Olczak, C. 2013, ApJ, 764, 146

Goldreich, P. \& Ward, W. 1973, ApJ, 183, 1051

Jacquet, E., Balbus, S., \& Latter, H. 2011, MNRAS, 415, 3591

Johansen, A., Youdin, A. N., \& Lithwick, Y. 2012, A\&A, 537, A125

Lee, A. T., Chiang, E. I., Asay-Davis, X., \& Barranco, J. 2010a, ApJ, 718, 1367

Lee, A. T., Chiang, E. I., Asay-Davis, X., \& Barranco, J. 2010b, ApJ, 725, 1938

Michikoshi, S., Kokubo, E., \& Inutsuka, S. 2012, ApJ, 746, 35

Shi, J.-M. \& Chiang, E. 2013, ApJ, 764, 20

Weidenschilling, S. J. 1980, Icarus, 44, 172

Weidenschilling, S. J. 2006, Icarus, 181, 572

Youdin, A. 2011, ApJ, 731, 99

Youdin, A. N. \& Goodman, J. 2005, ApJ, 620, 459

Zsom, A., Ormel, C. W., Dullemond, C. P., \& Henning, T. 2011, A\&A A, 534, A73

\section{Discussion}

NoRDLUND: If the mean magnetic field is strong enough, it can stabilize the flow against the Kelvin-Helmholtz instability.

ChIAng: Yes, magnetic tension can stabilize the flow against the KHI. But midplanes are poorly ionized and expected to be magnetically inactive.

FRASER: As clumps collapse by self-gravity, how much do particles grow by sticking, and can such growth protect the clump against turbulent disruption?

ChIAng: This needs to be further investigated. Relative velocities may be slower inside the clump, enabling growth beyond the usual limits.

GAIDos: Some planets are known to be formed around metal-poor stars.

ChIANG: Yes, but stellar metallicity and disk metallicity are distinct. What is relevant is the disk metallicity $Z_{\text {disk }}$ local to a given radius. Because solids drift radially (Andrews et al., this volume), $Z_{\text {disk }}$ can differ substantially from the host star metallicity. 